Água 



\section{Crise de abastecimento de água em São Paulo e falta de planejamento estratégico}

PEDRO LUIZ CÓRTES ${ }^{I}$, MAURO TORRENTE ${ }^{I I}$, Ailton PINTO AlVES FILHO ${ }^{I I I}$, MAURO SILVA RUIZ ${ }^{I V}$, ANTÓNIO JOSÉ GUERNER DIAS ${ }^{\mathrm{e}}$ e ROSELY RODRIGUES VI

\section{Introdução}

$\mathrm{D}$ ESDE O início de 2014, têm sido frequentemente divulgadas notícias relativas ao baixo nível pluviométrico em algumas das represas que abastecem a Região Metropolitana de São Paulo (RMSP). Ainda que essa redução da disponibilidade de água esteja sendo relacionada a um período de estiagem e de temperaturas muito acima das normais climatológicas para esta época do ano, ela é o reflexo da falta de planejamento estratégico que afeta o sistema de abastecimento da Região nos últimos dez anos. Embora essa escassez reflita um fenômeno que vem ocorrendo há alguns anos em outras regiões (Hadas; Gal, 2014; Zaimes; Emmanouloudis, 2012; Kummu et al., 2010), ela pode ser reduzida com um planejamento estratégico adequado que leve em consideração informações climáticas na concepção de ações. Há exemplos que ilustram essa possibilidade, como o estudo de cenários estratégicos empreendido por Hejazi et al. (2014) e que verificou a tendência de um aumento da escassez hídrica em diversos países, ou o estudo de Bolson e Broad (2013) sobre o gerenciamento de recursos hídricos na Flórida. Há também os estudos de Justes, Barberán e Farizo (2014) e Martin-Carrasco et al. (2013) na Espanha; Chun, Wheater e Onof (2013) no Reino Unido; e Wu e Wang (2010) no norte da China, para citar alguns exemplos, considerando diferentes cenários e escalas de abordagem.

O abastecimento público na Região é efetuado a partir de oito estações de tratamento de água, como na Tabela 1, sendo apenas uma delas (Guaraú) responsável pelo abastecimento de nove milhões de habitantes, fornecendo $45 \%$ da água consumida. Essa estação capta água do sistema denominado Cantareira, composto por quatro represas. A primeira delas fica entre os municípios de Bragança Paulista e Joanópolis e é alimentada pelos rios Jaguari e Jacareí, cujas nascentes se localizam no estado de Minas Gerais. A segunda represa armazena as águas do Rio Cachoeira do Piracaia, e a terceira, no município de Nazaré Paulista, retém as águas do Rio Atibainha. A quarta represa, denominada Engenheiro de Paiva Castro, está localizada entre os municípios de Mairiporã e Franco da Rocha na barra do Rio Juqueri. Todas essas represas estão interligadas e suas águas são tratadas pela ETA Guaraú, sendo destinadas ao abastecimento de cerca 
de nove milhões de pessoas das zonas Norte e Centro e parte das zonas Leste e Oeste da cidade de São Paulo, os municípios de Caieiras, Carapicuíba, Francisco Morato, Franco da Rocha, Osasco e São Caetano do Sul, e parte dos municípios de Barueri, Guarulhos, Santo André e Taboão da Serra, na RMSP (Chiodi; Sarcinelle; Uezu, 2013; Ribeiro, 2011; Pereira; Filho, 2009; Meyers, 2007).

Tabela 1 - Estações de tratamento de água na Região Metropolitana de São Paulo, respectiva produção e população atendida

\begin{tabular}{l|c|c}
\hline Sistema Produtor & Produção $\left(\mathrm{m}^{3} / \mathrm{s}\right)$ & População Atendida a $^{{ }^{2}}$ \\
\hline Alto Cotia & 1,2 & 0,41 \\
\hline Alto da Boa Vista & 14,0 & 3,70 \\
\hline Baixo Cotia & 0,9 & 0,42 \\
\hline Casa Grande & 4,0 & 2,06 \\
\hline Guaraú b & 33,0 & 9,00 \\
\hline Ribeirão da Estiva & 0,1 & 0,04 \\
\hline Rio Grande & 5,0 & 1,20 \\
\hline Taiaçupeba & 15,0 & 3,30 \\
\hline
\end{tabular}

Fonte: Sabesp (2014).

${ }^{a}$ Milhões de habitantes.

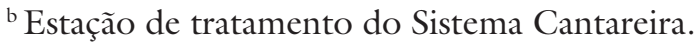

O crescimento da população na Região, demonstrado pela Figura 1, vem demandando investimentos na ampliação do sistema de abastecimento, com o aumento da capacidade das estações de tratamento, ou no desenvolvimento dos mananciais. Mesmo com o aporte na capacidade do sistema, tem ocorrido uma redução, ao longo dos anos, no volume de água per capita disponível nos mananciais para captação e no volume que as estações de tratamento de água (ETA) têm capacidade de tratar, conforme evidenciado pela Figura 2. Isso tem feito que o sistema trabalhe acima de sua capacidade operacional durante vários dias ao longo dos últimos anos, o que é demonstrado pela Figura 3. Embora essa capacidade operacional seja excedida ao longo de todo o ano, é nos meses mais quentes que essa frequência é maior, conforme indica a Figura 4, o que é consoante com a literatura (Aggarwal, et al., 2012; Connell-Buck et al., 2011; Hamlet, 2011; Praskievicz; Chang, 2009). Constata-se que a partir de dezembro até abril há maior incidência de dias em que o sistema de abastecimento trabalha acima de seu limite operacional. $\mathrm{O}$ mês de janeiro configura-se como exceção, pois há um grande fluxo de pessoas para o litoral e interior durante as férias escolares e as indústrias entram em período de férias coletivas. 


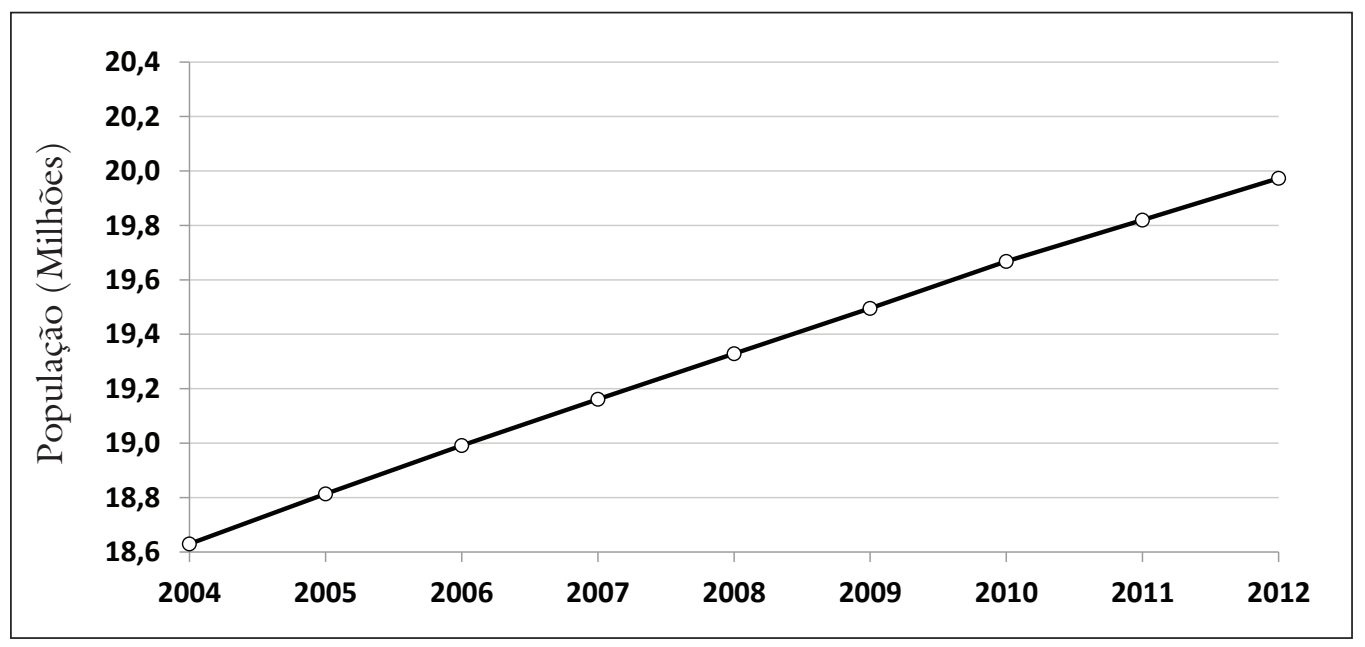

Fonte: Adaptado de IBGE (2013).

Figura 1 - Crescimento da população da RMSP entre 2004-2012.
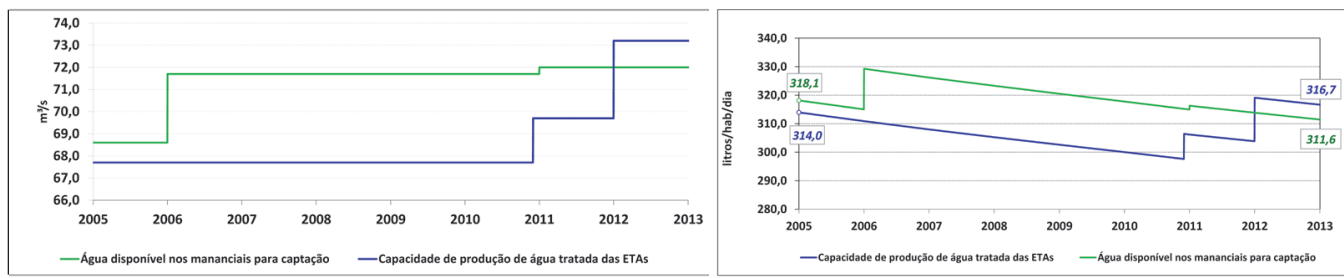

Volume de água disponível para captação e tratamento

Volume diário per capita de água para captação e tratamento

Fonte: Elaborado pelos autores a partir de Sabesp (2006, 2007, 2008, 2009, 2010, 2011, 2012).

Figura 2 - Evolução da capacidade do sistema de abastecimento da RMSP (2005 a 2012).

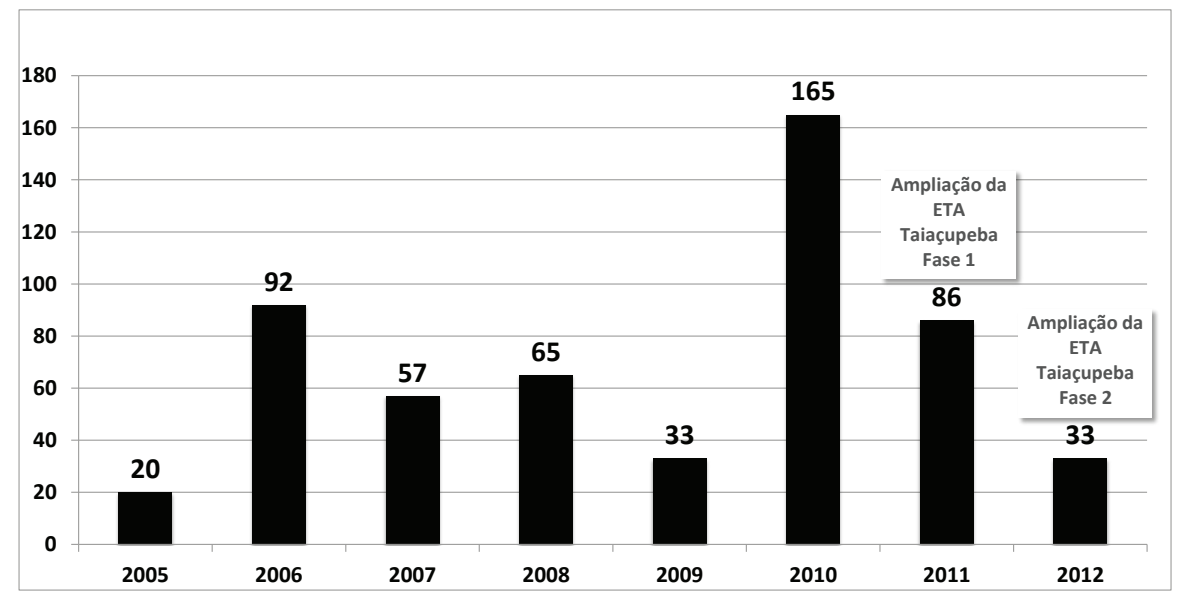

Fonte: Sabesp (2006, 2007, 2008, 2009, 2010, 2011, 2012, 2013a, 2013b).

Figura 3 - Total de dias em que a produção de água tratada excedeu o limite operacional. 


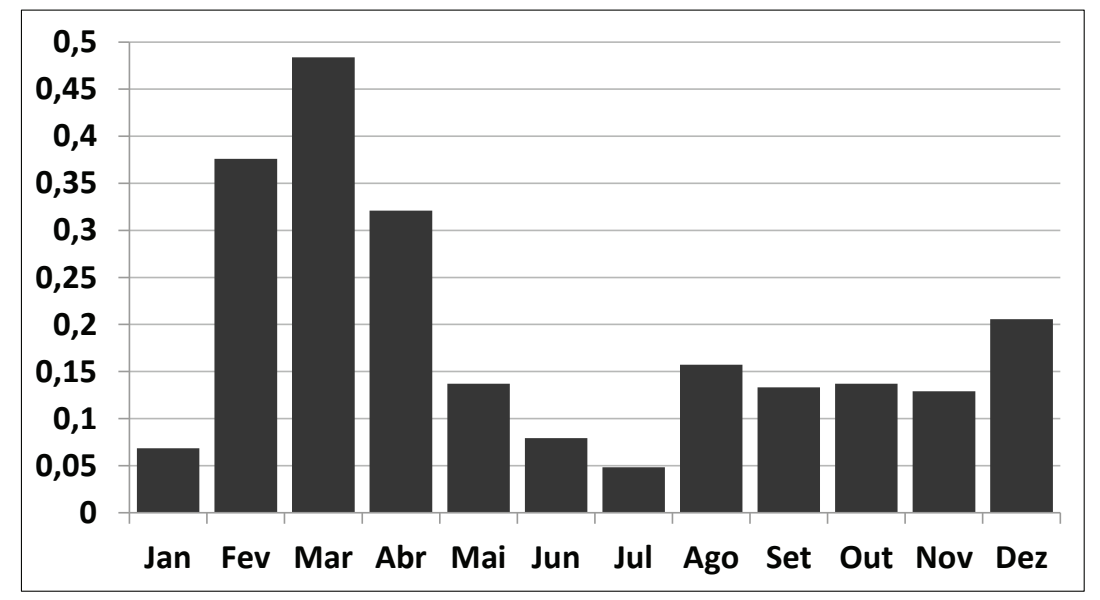

Fonte: Sabesp (2006, 2007, 2008, 2009, 2010, 2011, 2012, 2013a, 2013b).

Figura 4 - Percentual de dias em que a produção de água tratada superou o limite operacional, estratificados por mês (2005 a 2012).

Fica caracterizado que o sistema de abastecimento de água da Região não apenas sofre de uma deficiência crônica, com a perda de capacidade de atendimento sendo verificada pela redução dos volumes diários per capita, mas também fica mais suscetível a eventos climáticos como o ocorrido no verão de 20132014, com forte estiagem e elevadas temperaturas. A partir dessas considerações, surgiu a questão que norteou o desenvolvimento desta pesquisa "É possível utilizar informações climáticas na gestão estratégica do sistema de abastecimento da Região Metropolitana de São Paulo?”. A resposta a essa questão de pesquisa permitirá atingir o objetivo geral de "analisar o uso de variáveis climáticas no planejamento estratégico do abastecimento hídrico da RMSP". Para responder a questão e atender o objetivo traçado, foi elaborada uma estratégia de pesquisa que resultou no presente estudo, contando com o suporte de bibliografia especializada na definição das ações metodológicas (Gerring, 2012; Woodside, 2010; Kumar, 2010; YIN, 2008; Gerring, 2006; Yin, 2002), o qual foi iniciado por uma revisão da literatura com foco em estratégias ambientais, escassez hídrica resultante de ações humanas e influências climáticas.

\section{Revisão da literatura}

Os estudos sobre estratégias ambientais têm início da década de 1990 com trabalhos como o de Roome (1992) e Shrivastava e Hart (1995), sobre o desenvolvimento de estratégias ambientais nas organizações, ou a pesquisa de Green, Morton e New (1996), discorrendo sobre cadeias verdes de suprimentos. Prosseguiram com discussões sobre como as estratégias de responsabilidade socioambientais poderiam gerar valor para as empresas (Waddock; Graves, 1997) ou se constituir em vantagem competitiva (Sharma; Vredenburg, 1998). Na década seguinte, as estratégias ambientais foram ganhando espaço com a discussão sobre eco e socioeficiência e equidade ecológica (Dyllick; Hockerts, 2002), 
oferecendo orientação aos executivos e estrategistas de negócios (Olson, 2009). Mais recentemente, estudos de caso sobre estratégias ambientais começaram a ganhar destaque em publicações como o Journal of Business Strategy (Bonn; Fisher, 2011), assim como novos modelos para o desenvolvimento de estratégias ambientais de amplo alcance (Galpin; Whittington, 2012). Publicações mais específicas, como o periódico científico Business Strategy and the Environment, passaram a divulgar surveys com empresas (Ormazabal; Sarriegi, 2014) ou respostas estratégicas às mudanças climáticas (Galbreath, 2014).

As questões climáticas passaram, mais recentemente, a ocupar espaço significativo na concepção de estratégias organizacionais e políticas públicas, pois elas têm impacto direto na disponibilidade de água. Kummu et al. (2010) avaliaram a escassez de água entre os anos 0 e 2005 d.C. em 284 bacias hidrográficas. Tomando como base o indicador de Falkenmark (Falkenmark; Lundqvist; Widstrand, 1989), os autores constataram que em 1800 a escassez era um problema que pouco afetava as populações ao redor da Terra. Em 1900, 2\% da população mundial conviviam com escassez crônica de água, índice que passou a $9 \% \mathrm{em}$ 1960. Em 2005, 35\% da população mundial eram afetados por esse problema. Além das mudanças climáticas, houve um importante crescimento populacional e alterações ambientais promovidas pelo homem. Sob essa perspectiva, Cízková et al. (2013) analisaram as possíveis mudanças na Europa, incluindo escassez hídrica no sul, enchentes no oeste, enquanto o norte poderá reduzir a quantidade de matéria orgânica no solo. Como exemplo, é possível citar o Rio Ebro, um dos mais importantes da Espanha, que vem sofrendo com um decréscimo no fluxo de água nos rios, redução das médias de precipitação, aumento das temperaturas médias e do consumo (Milano et al., 2013).

Embora a compatibilização do consumo humano com as atividades agrícolas possa, em uma primeira análise, não ser um problema da RMSP, isso está longe de ser verdade, pois os rios que abastecem o Sistema Cantareira são compartilhados com a bacia dos rios Piracicaba, Capivari e Jundiaí (Bacia do PCJ). Essa bacia tem na agroindústria uma atividade importante, necessitando de água para a irrigação de culturas, processamento de alimentos e abastecimento urbano. Essa é uma situação que vem afetando diferentes países, conforme indicado pela pesquisa de Kummu et al. (2010), o que é corroborado pelo trabalho de Wada et al. (2013) ao avaliar que nos últimos cinquenta anos, a demanda de água mais do que dobrou, especialmente pelo incremento do consumo humano.

Ainda que esse problema esteja se intensificando, em parte como resultado do crescimento populacional e do comprometimento dos recursos hídricos existentes (Martin-Carrasco-Carrasco et al., 2013), nem sempre a população está consciente desse problema ou suficientemente informada sobre ele. Xiao (2013) lembra que na China as informações sobre a escassez e poluição das águas não são divulgadas ao público, repercutindo nos hábitos de consumo e na percepção sobre o real valor da água. Essa é uma situação que, segundo Côrtes (2013), se 
reflete na eficiência de políticas públicas e na conscientização ambiental. Kaldellis e Kondili (2007) relatam que o aumento da demanda para consumo humano e irrigação, redução das precipitações e aumento do uso de água subterrânea têm gerado escassez em determinadas regiões na Grécia, situação essa similar à verificada na RMSP, embora a população não tenha plena informação sobre isso (Ribeiro, 2011). Essa situação tem afetado de maneira indiscriminada os mais diversos países, como China (Wu; Wang, 2010); Austrália (Tapsuwan et al., 2014); Arábia Saudita (Ouda, 2014); Espanha (Milano et al., 2013); Estados Unidos (Gelcer et al., 2013); Chile (Meza, 2013; Núñez et al., 2013); Taiwan (Tsai; Elsberry, 2013), além de outras áreas na Europa e América do Norte (Hejazi et al., 2014).

Para mitigar esses problemas, diversas pesquisas têm sido realizadas como a adequação de sistemas de irrigação no norte da China (Wu; Wang, 2010), assunto também analisado por Alarcón, Garrido e Juana (2014), ou processos de dessalinização da água do mar na Espanha e em Israel (Hadas; Gal, 2014; Zarzo; Campos; Terrero, 2013). Há também estudos que avaliam a questão tarifária, como aquele desenvolvido por Justes, Barberán e Farizo (2014). Eles propõem que as tarifas sobre o consumo doméstico sejam pensadas tendo em consideração informações socioeconômicas dos moradores de cada residência, incluindo o padrão de consumo e comportamento, o que propiciaria melhores condições para o sucesso de campanhas de redução de consumo. Os dois temas - econômico e comportamental - também foram considerados, respectivamente, pelos estudos de Horne (2013) e Zaimes e Emmanouloudis (2012). Há também estudos sobre a reutilização de água (Atkinson, 2014) ou captação de água de chuva (Morales-Pinzón et al., 2014; Morales-Pinzón et al., 2012), buscando fontes alternativas que reduzam a dependência de sistemas convencionais de captação, tratamento e distribuição.

Outros estudos propõem a utilização de sistemas computacionais e algoritmos com modelagens específicas para ajudar na gestão de recursos hídricos (Ngoc; Hiramatsu; Harada, 2014; Xiao-Jun et al., 2014). Em abordagem similar, Kangrang et al. (2013) e Kangrang e Lokham (2013) indicam o uso de técnicas para melhorar o gerenciamento em longo prazo dos reservatórios de água. Martin-Carrasco et al. (2013), por sua vez, sugeriram índices na estimativa de escassez. Ainda considerando a utilização de sistemas de modelagem, há pesquisas sobre a utilização de parâmetros climáticos para avaliação dos padrões de chuva no Reino Unido (Chun; Wheater; Onof, 2013) ou no comportamento hídrico de sub-bacias do Rio Colorado (Kalra et al., 2013). Outra possibilidade, conforme indicado por Bolson e Broad (2013), é a adoção de informações sobre o clima em sistemas de apoio à decisão no gerenciamento de recursos hídricos, conforme efetuado pelo South Florida Water Management District.

Tendo em perspectiva a utilização de parâmetros climáticos, é necessário considerar que o clima na região Sul do Brasil, assim como em parte da região 
Sudeste, é influenciado pelo fenômeno natural conhecido como El Niño Oscilação Sul ou Enso, derivado de sua sigla em inglês adotada internacionalmente (Gunn, 2010). Ele consiste em um aquecimento das águas do Oceano Pacífico na costa do Peru, e a situação oposta (resfriamento das águas na mesma região) é denominada La Niña, ambos apresentando períodos de alternância e repercutindo no clima mundial. Com o El Niño, por exemplo, há um aumento dos valores pluviométricos na região Sul do Brasil durante o verão, enquanto com o La Niña ocorrem períodos de estiagem para o mesmo período e região (Feliciani et al., 2013). Influência similar, embora com menor intensidade, verifica-se na porção centro-este do estado de São Paulo (Santos et al., 2012).

Na região Nordeste ocorre o oposto da região Sul, com o El Niño sendo associado a uma tendência de estiagem e o La Niña, a períodos de maior pluviosidade (Kwon et al., 2012). A influência do fenômeno também é verificada em Minas Gerais (Mello et al., 2008), na disponibilidade hídrica do Rio Grande do Sul (Teixeira; Satyamurty, 2011; Campos; Silva, 2010) e no Paraná (Baú; De Azevedo; Bresolin, 2013). Estudo empreendido por Silva Dias et al. (2013) relata a influência desse sistema na RMSP. Suas ações igualmente se manifestam no Chile (Arumí et al., 2013; Meza, 2013), Taiwan (Tsai; Elsberry, 2013), sul da China (Zhang et al., 2013) e Califórnia (Gelcer et al., 2013).

\section{Metodologia}

Tendo como base a questão de pesquisa "É possível utilizar informações climáticas na gestão estratégica do sistema de abastecimento da Região Metropolitana de São Paulo?" e o objetivo de "analisar o uso de variáveis climáticas no planejamento estratégico do abastecimento hídrico da Região”, foi concebida uma estratégia de pesquisa que resultou no presente trabalho, caracterizado como um estudo de caso sobre o abastecimento de água na Região, apoiado em dados quantitativos e documentos de fontes primárias (Companhia de Saneamento Básico do Estado de São Paulo - Sabesp e Estação Meteorológica do Instituto Astronômico e Geofísico da Universidade de São Paulo - IAG/USP) e secundárias (Climate Prediction Center da National Oceanic and Atmospheric Administration - CPC/NOAA). Essa estratégia contou com bibliografia especializada na definição das ações metodológicas (Gerring, 2012; Woodside, 2010; Kumar, 2010; Yin, 2008; Gerring, 2006), no tratamento estatístico dos dados (Hair Jr. et al., 2013; Gamst; Meyers; Guarino, 2008; Landau; Everitt, 2004) e em sua interpretação (Xu; Sano; Nakatsuka, 2013; Barry; Chorley, 2010; Gergis; Fowler, 2009).

\section{Resultados da pesquisa e implicações decorrentes}

Embora seja presumível que ocorra maior consumo de água com a elevação da temperatura, é necessário considerar que a Região Metropolitana de São Paulo conta com uma série de atividades econômicas, especialmente de natureza industrial, com um uso constante de água que não é afetado diretamente pela temperatura. Em que pese o processo de desindustrialização verificado no esta- 
do (Cleps, 2003; Negri, 1996), essa atividade responde por 23,53\% do Produto Interno Bruto da RMSP (Seade, 2014) e demanda um uso intensivo de água (Alkaya; Demirer, 2014; Vos; Boelens, 2014; Wojdalski et al., 2013). Ponderou-se como necessário verificar qual a influência da temperatura no consumo de água na Região, aspecto considerado fundamental no planejamento estratégico do sistema de abastecimento. Foram considerados dados primários sobre a produção de água nas oito estações de tratamento de água (Tabela 1), para cada dia do período compreendido entre 2005 e 2012. Esses dados foram correlacionados com a temperatura máxima registrada pela Estação Meteorológica do Instituto Astronômico e Geofísico da Universidade de São Paulo (IAG/USP), gerando a Figura 5. Para esse conjunto de dados foi calculado o coeficiente de correlação de Pearson (Mooi; Sarstedt, 2011), obtendo-se o valor de 0,491334, considerado moderado (Dancey; Reidy, 2006; Landau; Everitt, 2004), o que indica que o consumo de água é influenciado parcialmente pelo aumento da temperatura.

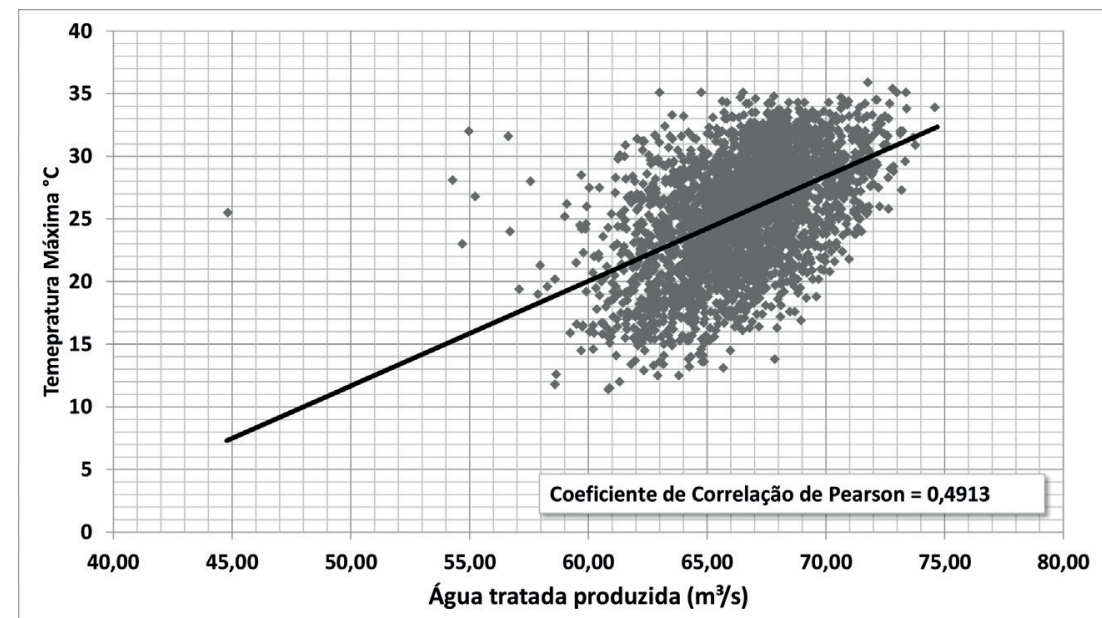

Fonte: Elaborado a partir de Sabesp $(2006,2007,2008,2009,2010,2011,2012,2013)$ e IAG-USP (2014).

Figura 5 - Produção de água x temperatura máxima na RMSP (2005 a 2012).

Para melhor compreender esse comportamento, foi calculado o coeficiente de correlação de Pearson para faixas específicas de temperatura, conforme disponível na Tabela 2. Partiu-se da suposição de que essa correlação ficaria mais forte à medida que a temperatura subisse, o que seria condizente com os estudos de Milano et al. (2013) e Slavíková et al. (2013). Essa suposição se baseia no fato de que o consumo basal, especialmente decorrente das atividades industriais, seria diluído com o crescimento do consumo decorrente do aumento da temperatura. Isso tornaria as correlações mais fortes para temperaturas mais elevadas. Verificou-se o oposto, conforme demonstrado pela Tabela 2 e pela Figura 6, com as correlações ficando mais fracas com o aumento da temperatura, o que 
indica uma perda de elasticidade do sistema de abastecimento para temperaturas mais elevadas. Analisando a Figura 3, constata-se que em 551 dias do período considerado (2005-2012) o sistema operou além de sua capacidade operacional, o que demonstra uma demanda elevada, incapaz de ser adequadamente atendida. A Figura 4, por sua vez, evidencia que esse aumento de consumo ocorre exatamente nos meses mais quentes, à exceção de janeiro conforme explicado anteriormente. Todas essas informações ratificam que há uma demanda potencial ampliada com o aumento da temperatura, mas também há uma incapacidade de o sistema atender adequadamente a essa mesma demanda.

Tabela 2 - Correlações entre a produção de água e temperatura máxima na RMSP (2005 a 2012)

\begin{tabular}{c|c}
\hline Fixa de Temperatura $\left({ }^{\circ} \mathrm{C}\right)$ & $\begin{array}{c}\text { Coeficiente de Correlação } \\
\text { de Pearson }\end{array}$ \\
\hline Todos os valores & 0,491334 \\
\hline $\mathrm{t}<=15$ & 0,349983 \\
\hline $15<\mathrm{t}<=20$ & 0,270814 \\
\hline $20<\mathrm{t}<=25$ & 0,109992 \\
\hline $25<\mathrm{t}<=30$ & 0,159600 \\
\hline $30<\mathrm{t}<=35$ & 0,167420 \\
\hline
\end{tabular}

Fonte: Elaborado a partir de Sabesp $(2006,2007,2008,2009,2010,2011,2012,2013 a)$ e IAG-USP (2014).

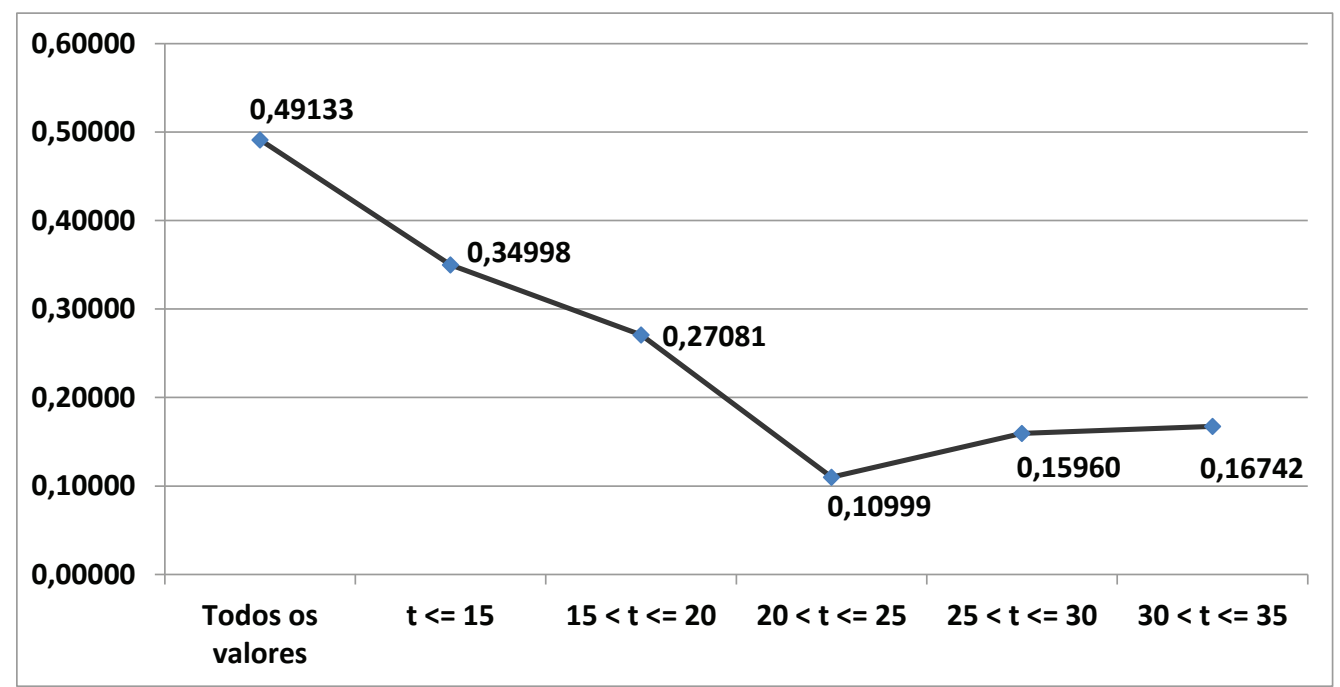

Fonte: Elaborado a partir de Sabesp $(2006,2007,2008,2009,2010,2011,2012,2013 a)$ e IAG-USP (2014).

Figura 6 - Correlações “produção de água versus temperatura máxima” na RMSP. 
Em decorrência dessa falta de elasticidade, o sistema de abastecimento da RMSP apresenta baixa capacidade de assimilação de eventos climáticos como o ocorrido no verão 2013-2014, com uma seca prolongada e altas temperaturas. Comparativamente, os meses de janeiro e fevereiro de 2014 foram mais quentes do que no mesmo período em 2013 (Figura 7), ampliando o consumo de água. No verão 2013-2014, esse maior consumo e a seca prolongada promoveram uma queda do nível das represas do Sistema Cantareira, responsável por 45,8\% da água tratada da RMSP, como indica a Figura 8. Analisando os níveis do sistema desde 2004, constata-se que o período de recarga do sistema vai de outubro a março do ano subsequente. Desde 2011, entretanto, verifica-se uma tendência de redução na recomposição do nível do sistema, representada pela reta $\mathrm{AB}$ na Figura 8. Essa tendência é reforçada pela Figura 9, que evidencia que ao longo do segundo semestre de 2013 não houve indício de que haveria uma elevação do nível d'água no sistema, informação essa que poderia ter sido utilizada para a concepção de estratégias preventivas (Galbreath, 2014; Galpin; Whittington, 2012).
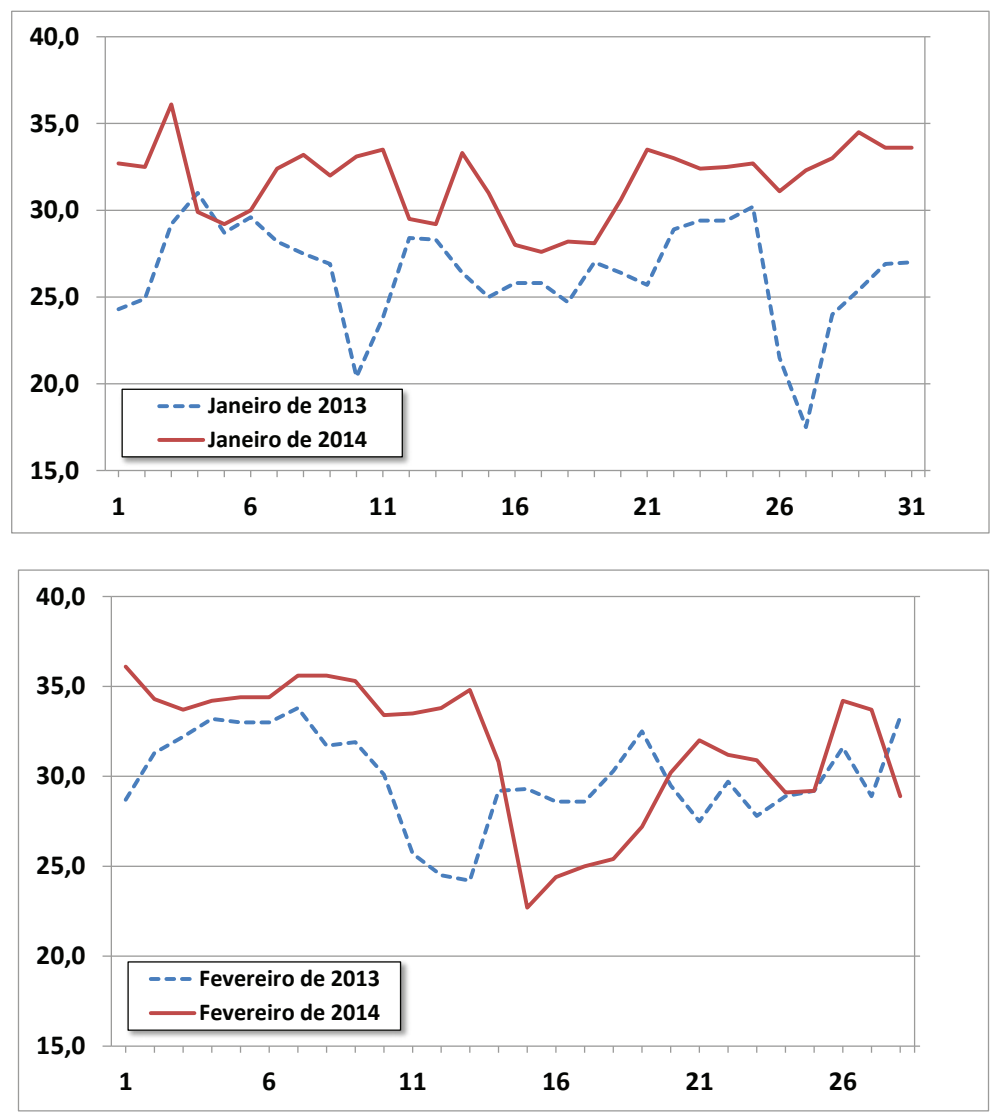

Fonte: IAG-USP (2014).

Figura 7 - Comparativo entre as temperaturas máximas na RMSP para os meses de janeiro de fevereiro de 2013 e 2014. 
Para melhor avaliar a influência climática, foram utilizados dados primários coletados pela Estação Meteorológica do Instituto Astronômico e Geofísico da Universidade de São Paulo (IAG/USP) e dados secundários fornecidos pelo Climate Prediction Center da National Oceanic and Atmospheric Administration (CPC/NOAA) para a elaboração da Tabela 3. Nela, é possível verificar as médias pluviométricas mensais, semestrais e no período de recarga do Sistema Cantareira, de 1950 a 2013, assim como a indicação da fase predominante do fenômeno Enso.

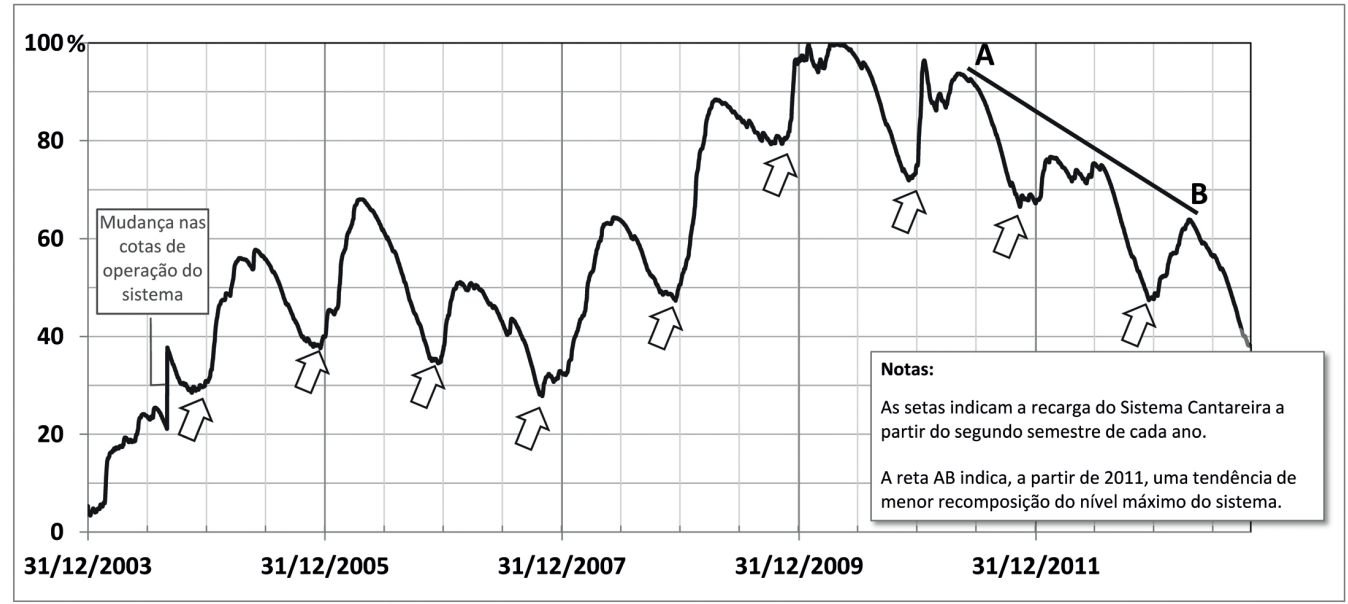

Fonte: Adaptado de Agência Nacional de Aguas (2014, p.9).

Figura 8 - Evolução do volume útil do Sistema Cantareira entre 2004 e 2014.

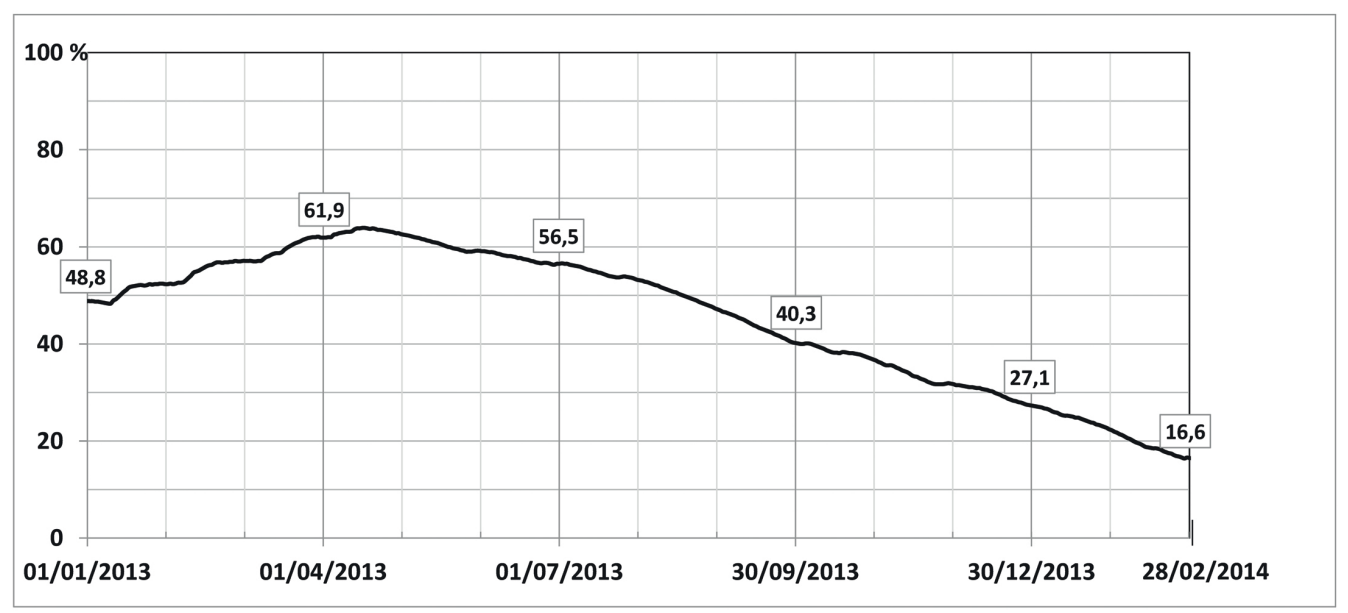

Fonte: Agência Nacional de Águas (2014, p.9).

Figura 9 - Evolução do volume útil do Sistema Cantareira entre jan. 2013 e fev. de 2014. 
Tabela 3 - Médias pluviométricas ( $\mathrm{mm}$ ) mensais, semestrais e no período de recarga (1950-2013)

\begin{tabular}{|c|c|c|c|c|c|c|c|c|c|c|c|c|c|c|c|}
\hline \multirow{2}{*}{ Fase } & \multicolumn{15}{|c|}{ Período } \\
\hline & Jan & Fev & Mar & Abr & Mai & Jun & Jul & Ago & Set & Out & Nov & Dez & $1^{\circ}$ Semestre ${ }^{b}$ & $2^{\circ}$ Semestre $^{\mathrm{b}}$ & Recarga $\mathrm{b}, \mathrm{c}$ \\
\hline El Niño & 238,7 & 225,4 & 177,7 & 118,1 & 96,4 & 73,2 & 53,3 & 39,9 & 80,2 & 119,8 & 154,3 & 181,4 & 945,5 & 637,6 & 1102 \\
\hline Neutra & 209,1 & 218,8 & 163,8 & 80,5 & 62,3 & 47,8 & 45,4 & 38,8 & 76,1 & 144,5 & 132,9 & 193,7 & 832,2 & 600,1 & 1097 \\
\hline La Niña & 254,8 & 222,9 & 169,1 & 81,4 & 60,3 & 44,9 & 44,5 & 27,1 & 72,8 & 121,7 & 106,7 & 199,5 & 826,4 & 573,7 & 1088 \\
\hline Geral a & 239,6 & 221,7 & 167,9 & 87,1 & 68,3 & 52,7 & 46,5 & 36,8 & 76,6 & 128,0 & 130,8 & 188,9 & 845,2 & 607,6 & 1074 \\
\hline
\end{tabular}

Fontes: Precipitação pluviométrica IAG/USP e Sistema El Niño - Oscilação Sul CPC/NOAA. a Consolidado para o período indicado, sem distinção da fase do Sistema El Niño - Oscilação Sul.

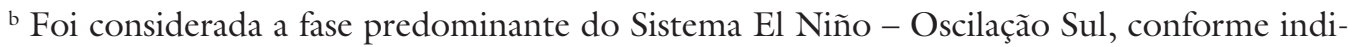
cado em cada linha.

${ }^{\mathrm{c}}$ Meses considerados: outubro, novembro, dezembro, janeiro, fevereiro e março.

Analisando a Tabela 3, é possível perceber que os semestres e períodos de recarga com predominância da fase El Niño apresentam maior pluviosidade, o que está de acordo com os estudos empreendidos por Santos et al. (2012) para o interior do estado, e Silva Dias et al. (2013) para a cidade de São Paulo. Outro ponto de destaque é que na fase El Niño o período de chuvas não apresenta uma queda brusca a partir de março-abril, se estendendo pelos meses de maio e junho, o que potencialmente amplia a recarga do Sistema Cantareira (Figura 10). Essas informações, juntamente com a evolução recente do fenômeno Enso, indicam que o período de maior decréscimo no volume do Sistema Cantareira (representado pela reta $\mathrm{AB}$, na Figura 8) ocorre sob a predominância de uma fase neutra, exatamente como verificado no período de abril de 2012 a abril de 2013. Considerando que os prognósticos sobre o fenômeno Enso são efetuados por diversos serviços meteorológicos com pelo menos seis meses de antecedência, essa informação já indicava em meados de 2013 que o Sistema Cantareira não conseguiria recompor o seu nível no segundo semestre desse mesmo ano. Caso essa informação fosse utilizada pelo Governo do Estado e pela Companhia de Saneamento Básico do Estado de São Paulo (Sabesp), seria possível o desenvolvimento de estratégias de enfrentamento, evitando seu agravamento no segundo semestre de 2013. 


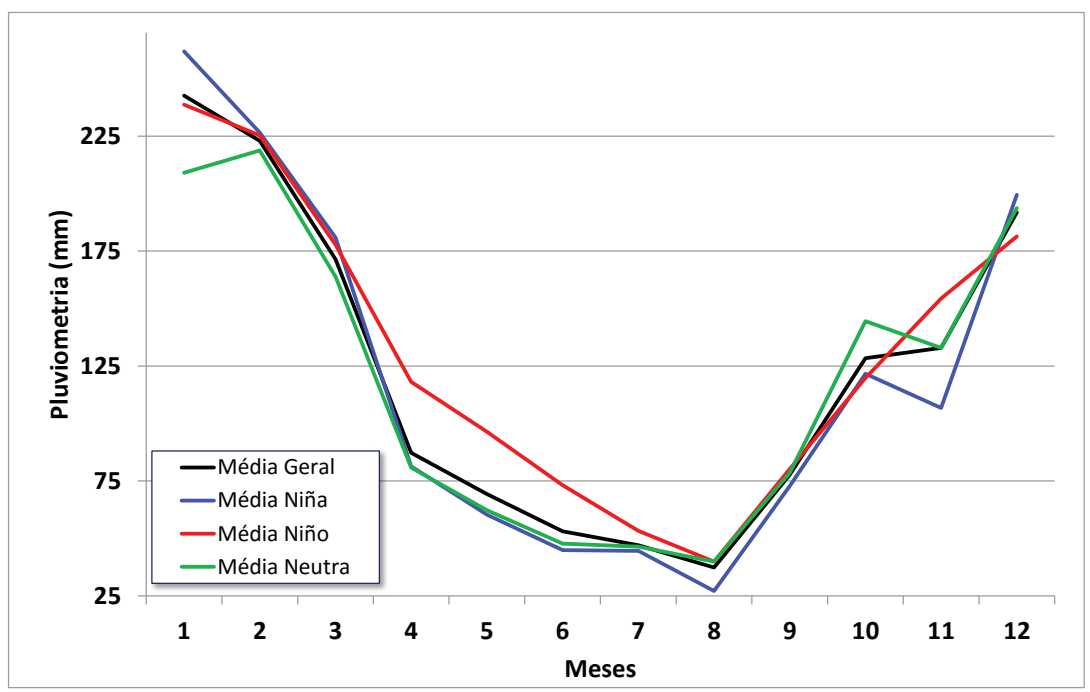

Fonte: Elaborado a partir de médias mensais fornecidas pelo IAG/USP.

Figura 10 - Médias pluviométricas (mm) mensais (1950-2013).

\section{Conclusões}

Este trabalho averiguou a possibilidade de utilizar informações climáticas na gestão estratégica do sistema de abastecimento da Região Metropolitana de São Paulo. Foram tratados dados obtidos a partir de fontes primárias e secundárias, correlacionando temperatura com o consumo de água e verificando como eventos climáticos associados ao fenômeno Enso repercutem na recomposição do Sistema Cantareira. Constatou-se que o uso de informações ambientais pode desempenhar um papel estratégico para a gestão do abastecimento urbano. $\mathrm{O}$ fenômeno Enso tem suas ações prognosticadas com pelo menos seis meses de antecedência, o que possibilita antever os impactos decorrentes e determinar o desenvolvimento de estratégias mitigadoras. Diante dessa circunstância, é proposto o seguinte conjunto de ações que têm impacto em diferentes horizontes temporais: i) utilizar efetivamente as informações e prognósticos climáticos na gestão estratégica da distribuição de água na RMSP (Bolson; Broad, 2013); ii) promover o acesso da população às informações ambientais, comunicando os prognósticos climáticos e sua repercussão para o abastecimento urbano (Côrtes, 2013 ); iii) desenvolver políticas permanentes que incentivem o uso racional da água e promovam fontes alternativas (isto é, água de reúso, captação de água de chuva), reduzindo a pressão sobre o sistema de abastecimento (Atkinson, 2014; Morales-Pinzón et al., 2012); iv) alterar a política tarifária, implantando um sistema que incentive a economia de água (Justes; Barberán; Farizo, 2014; Horne, 2013; Zaimes; Emmanouloudis, 2012), levando em consideração situações contingenciais que tornem automática a cobrança de taxas adicionais para consumo excessivo em caso de redução anormal do nível dos mananciais (ou de prognósticos que indiquem essa possibilidade). 


\section{Referências}

AGÊNCIA NACIONAL DE ÁGUAS. Boletim de Monitoramento dos Reservatórios do Sistema Cantareira. ANA - Agência Nacional de Águnas. Brasília, 2014. p.12.

AGGARWAL, R. M. et al. How do variations in Urban Heat Islands in space and time influence household water use? the case of Phoenix, Arizona. Water Resources Research, v.48, 2012 .

ALARCÓN, J.; GARRIDO, A.; JUANA, L. Optimal water allocation in shortage situations as applied to an irrigation community. Journal of Irrigation and Drainage Engineering, v.140, 2014.

ALKAYA, E.; DEMIRER, G. N. Sustainable textile production: A case study from a woven fabric manufacturing mill in Turkey. Journal of Cleaner Production, v.65, p.595$603,2014$.

ARUMÍ, J. L. et al. Effect of drought on groundwater in a Chilean irrigated valley. Proceedings of the Institution of Civil Engineers: Water Management, v.166, p.231-41, 2013.

ATKINSON, W. Reusable waters. Pollution Engineering, v.46, p.23-5, 2014.

BARRY, R. G.; CHORLEY, R. J. Atmosphere, weather and climate. New York: Routledge, 2010.

BAÚ, A. L.; DE AZEVEDO, C. A.; BRESOLIN, A. A. Modeling of daily intra-annual precipitation of the Paraná III Basin associated with events ENSO. Revista Brasileira de Engenharia Agricola e Ambiental, v.17, p.883-91, 2013.

BOLSON, J.; BROAD, K. Early adoption of climate information: Lessons learned from south florida water resource management. Weather, Climate, and Society, v.5, p.266-81, 2013.

BONN, I.; FISHER, J. Sustainability: The missing ingredient in strategy. Journal of Business Strategy, v.32, p.5-14, 2011.

CAMPOS, C. R. D.; SILVA, M. V. D. Impact of weather systems in the hydrological regime of Rio Grande do Sul in 2006. Revista Brasileira de Geofisica, v.28, p.121-36, 2010.

CHIODI, R. E.; SARCINELLE, O.; UEZU, A. Management of water resources in the Cantareira water producer system area: A look at the rural context. Revista Ambiente e Água, v.8, p.151-65, 2013.

CHUN, K. P.; WHEATER, H.; ONOF, C. Prediction of the impact of climate change on drought: an evaluation of six UK catchments using two stochastic approaches. Hydrological Processes, v.27, p.1600-14, 2013.

CÍZKOVÁ, H. et al. Actual state of European wetlands and their possible future in the context of global climate change. Aquatic Sciences, v.75, p.3-26, 2013.

CLEPS, G. D. G. A Desconcentração industrial no Estado de São Paulo e a expansão do comércio e do setor de serviços. Caminhos de Geografia, v.4, n.9, p.66-89, 2003.

CONNELL-BUCK, C. R. et al. Adapting Californias water system to warm vs. dry climates. Climatic Change, v.109, p.133-49, 2011.

CÔRTES, P. L. Conception and development of a system used to organize and facili- 
tate access to environmental information. JISTEM - Journal of Information Systems and Technology Management, v.10, n.1, p.161-76, 2013.

DANCEY, C. P.; REIDY, J. Estatística sem matemática para psicologia. Porto Alegre: Artmed, 2006.

DYLLICK, T.; HOCKERTS, K. Beyond the business case for corporate sustainability. Business Strategy and the Environment, v.11, p.130-41, 2002.

FALKENMARK, M.; LUNDQVIST, J.; WIDSTRAND, C. Macro-scale water scarcity requires micro-scale approaches. Aspects of vulnerability in semi-arid development. $\mathrm{Na}$ tural Resources Forum, v.13, p.258-67, 1989.

FELICIANI, A. V. et al. Analysis of the flow regime of the Jaguari river by means of stochastic models. Espacios, v.34, 2013.

GALBREATH, J. Climate change response: Evidence from the margaret river wine region of Australia. Business Strategy and the Environment, v.23, p.89-104, 2014.

GALPIN, T.; WHITTINGTON, J. L. Sustainability leadership: From strategy to results. Journal of Business Strategy, v.33, p.40-8, 2012.

GAMST, G.; MEYERS, L. S.; GUARINO, A. J. Analysis of variance designs. Cambridge: Cambridge University Press, 2008.

GELCER, E. et al. Effects of El Niño Southern Oscillation on the space-time variability of Agricultural Reference Index for Drought in midlatitudes. Agricultural and Forest Meteorology, v.174-75, p.110-28, 2013.

GERGIS, J. L.; FOWLER, A. M. A history of ENSO events since A.D. 1525: Implications for future climate change. Climatic Change, v.92, p.343-87, 2009.

GERRING, J. Case study research: principles and practices. Cambridge: Cambridge University Press, 2006. 278p.

GERRING, J. Social science methodology: a unified framework (Strategies for Social Inquiry). Cambridge: Cambridge University Press, 2012.

GREEN, K.; MORTON, B.; NEW, S. Purchasing and environmental management: interactions, policies and opportunities. Business Strategy and the Environment, v.5, p.188-97, 1996.

GUNN, A. M. A Student Guide to Climatte and Weather. Santa Barbara: Greenwood, 2010. v.1 Weather Extremes.

HADAS, E.; GAL, Y. Inter-sector water allocation in Israel, 2011-2050: Urban consumption versus farm usage. Water and Environment Journal, v.28, p.63-71, 2014.

HAIR JUNIOR, J. F. et al. Multivariate Data Analysis. s. 1.: Pearson, 2013.

HAMLET, A. F. Assessing water resources adaptive capacity to climate change impacts in the Pacific Northwest Region of North America. Hydrology and Earth System Sciences, v.15, p.1427-43, 2011.

HEJAZI, M. et al. Long-term global water projections using six socioeconomic scenarios in an integrated assessment modeling framework. Technological Forecasting and Social Change, v.81, p.205-26, 2014.

HORNE, J. Economic approaches to water management in Australia. International Journal of Water Resources Development, v.29, p.526-43, 2013. 
IAG-USP. Relatório de Dados Climatológicos da Cidade de São Paulo - (período 1950 - 2014). São Paulo. 2014.

IBGE. Estimativas da população residente nos municípios brasileiros com data de referência de $1^{\circ}$ de julho de 2013. Brasília. 2013.

JUSTES, A.; BARBERÁN, R.; FARIZO, B. A. Economic valuation of domestic water uses. Science of the Total Environment, v.472, p.712-18, 2014.

KALDELLIS, J. K.; KONDILI, E. M. The water shortage problem in the Aegean archipelago islands: cost-effective desalination prospects. Desalination, v.216, p.123-38, 2007.

KALRA, A. et al. Using large-scale climatic patterns for improving long lead time streamflow forecasts for Gunnison and San Juan River Basins. Hydrological Processes, v.27, p.1543-59, 2013.

KANGRANG, A. et al. Expert participation with optimization technique for improving optimal rule curves of reservoir. Bulgarian Journal of Agricultural Science, v.19, p.1140-47, 2013.

KANGRANG, A.; LOKHAM, C. Optimal reservoir rule curves considering conditional ant colony optimization with simulation model. Journal of Applied Sciences, v.13, p.154-60, 2013.

KUMAR, R. Research methodology: a step-by-step guide for beginners. Thousand Oaks: SAGE Publications, 2010.

KUMMU, M. et al. Is physical water scarcity a new phenomenon? Global assessment of water shortage over the last two millennia. Environmental Research Letters, v.5, 2010.

KWON, H. et al. Uncertainty assessment of hydrologic and climate forecast models in Northeastern Brazil. Hydrological Processes, v.26, p.3875-85, 2012.

LANDAU, S.; EVERITT, B. S. A handbook of statistical analyses using SPSS. Boca Raton: Chapman \& Hall/CRC Press, 2004.

MARTIN-CARRASCO, F. et al. Diagnosing causes of water scarcity in complex water resources systems and identifying risk management actions. Water Resources Management, v.27, p.1693-705, 2013.

MELLO, C. R. et al. Development and application of a simple hydrologic model simulation for a Brazilian headwater basin. Catena, v.75, p.235-47, 2008.

MEYERS, L. Brazilian water utility keeps up with fast-growing population demands. Water and Wastewater International, v.22, p.42-3, 2007.

MEZA, F. J. Recent trends and ENSO influence on droughts in Northern Chile: an application of the Standardized Precipitation Evapotranspiration Index. Weather and Climate Extremes, v.1, p.51-8, 2013.

MILANO, M. et al. Modeling the current and future capacity of water resources to meet water demands in the Ebro basin. Journal of Hydrology, v.500, p.114-26, 2013.

MOOI, E.; SARSTEDT, M. A concise guide to market research. Heidelberg: Springer, 2011.

MORALES-PINZÓN, T. et al. Potential of rainwater resources based on urban and social aspects in Colombia. Water and Environment Journal, v.26, p.550-9, 2012. 
MORALES-PINZÓN, T. et al. Financial and environmental modelling of water hardness - Implications for utilising harvested rainwater in washing machines. Science of the Total Environment, v.470-1, p.1257-71, 2014.

NEGRI, B. Concentração e desconcentração industrial em São Paulo (1880 1990). Campinas: Editora da Unicamp, 1996.

NGOC, T. A.; HIRAMATSU, K.; HARADA, M. Optimizing the rule curves of multi-use reservoir operation using a genetic algorithm with a penalty strategy. Paddy and Water Environment, v.12, p.125-37, 2014.

NÚÑEZ, J. et al. Influence of Pacific Ocean multidecadal variability on the distributional properties of hydrological variables in north-central Chile. Journal of Hydrology, v.501, p.227-40, 2013.

OLSON, E. G. Business as environmental steward: The growth of greening. Journal of Business Strategy, v.30, p.4-13, 2009.

ORMAZABAL, M.; SARRIEGI, J. M. Environmental management evolution: Empirical evidence from Spain and Italy. Business Strategy and the Environment, v.23, p.73-88, 2014.

OUDA, O. K. Water demand versus supply in Saudi Arabia: current and future challenges. International Journal of Water Resources Development, v.30, p.335-44, 2014.

PEREIRA, V. R.; FILHO, J. T. Identifying susceptible areas to erosive processes in the Cantareira System, based on different scenarios. Acta Scientiarum - Agronomy, v.31, p.155-63, 2009.

PRASKIEVICZ, S.; CHANG, H. Identifying the relationships between urban water consumption and weather variables in Seoul, Korea. Physical Geography, v.30, p.324-37, 2009.

RIBEIRO, W. C. Water supply and water stress in the Metropolitan Region of São Paulo. Estudos Avançados, v.25, n.71, p.119-33, 2011.

ROOME, N. Developing environmental management strategies. Business Strategy and the Environment, v.1, p.11-24, 1992.

SABESP. Securities and Exchange Commission dos Estados Unidos. Formulário 20F 2005. São Paulo. 2006.

SABESP. IAN - Informações Anuais à Comissão de Valores Mobiliários. São Paulo. 2007.

SABESP. Securities and Exchange Commission dos Estados Unidos. Formulário 20F 2007. São Paulo. 2008.

SABESP. Formulário 20-F 2008. Relatório anual de acordo com o artigo 13 ou 15(d) da Lei de Valores Imobiliários de 1934 referente ao exercício social encerrado em 31 de dezembro de 2008. São Paulo. 2009.

SABESP. Formulário 20-F 2009. Relatório anual de acordo com o artigo 13 ou 15(d) da Lei de Valores Imobiliários de 1934 referente ao exercício social encerrado em 31 de dezembro de 2009. São Paulo. 2010.

SABESP. Formulário 20-F 2010. Relatório anual de acordo com o artigo 13 ou 15(d) da Lei de Valores Imobiliários de 1934, referente ao exercício encerrado em 31 de dezembro de 2010. São Paulo. 2011. 
SABESP. Formulário 20-F 2011. Relatório anual de acordo com o artigo 13 ou 15(d) da Lei de Valores Imobiliários de 1934 referente ao exercício social encerrado em 31 de dezembro de 2011. São Paulo. 2012.

SABESP. Relatório interno sobre produção diária de água entre 2004 e 2012 para a Região Metropolitana de São Paulo. São Paulo. 2013.

SABESP. Formulário 20-F 2012. Relatório anual de acordo com o artigo 13 ou 15(d) da Lei de Valores Imobiliários de 1934 referente ao exercício social encerrado em 31 de dezembro de 2012. São Paulo. 2013a.

SABESP. Produção de água na Região Metropolitana de São Paulo: 2004 a 2012. São Paulo. 2013b.

SABESP. Tratamento de água: complexo metropolitano. São Paulo. 2014.

SANTOS, C. A. C. D. et al. Variability of extreme climate indices at Rio claro, São Paulo, Brazil. Revista Brasileira de Meteorologia, v.27, n.4, p.395-400, 2012.

SEADE. Perfil Regional. Fundação Sistema Estadual de Análise de Dados, 26 abril 2014. Disponivel em: <http://www.seade.gov.br/produtos/perfil_regional/index. php>.

SHARMA, S.; VREDENBURG, H. Proactive corporate environmental strategy and the development of competitively valuable organizational capabilities. Strategic Management Journal, v.19, p.729-53, 1998.

SHRIVASTAVA, P.; HART, S. Creating sustainable corporations. Business Strategy \& the Environment, v.4, p.154-65, 1995.

SILVA DIAS, M. A. et al. Changes in extreme daily rainfall for São Paulo, Brazil. Climatic Change, v.116, p.705-22, 2013.

SLAVÍKOVÁ, L. et al. Impacts of Climate Variables on Residential Water Consumption in the Czech Republic. Water Resources Management, v.27, p.365-79, 2013.

TAPSUWAN, S. et al. Adapting to less water: household willingness to pay for decentralised water systems in urban Australia. Water Resources Management, v.28, p.111125,2014 .

TEIXEIRA, M. S.; SATYAMURTY, P. Trends in the frequency of intense precipitation events in southern and southeastern Brazil during 1960-2004. Journal of Climate, v.24, p.1913-21, 2011.

TSAI, H.; ELSBERRY, R. L. Opportunities and challenges for extended-range predictions of tropical cyclone impacts on hydrological predictions. Journal of Hydrology, v.506, p.42-54, 2013.

VOS, J.; BOELENS, R. Sustainability standards and the water question. Development and Change, v.45, p.205-30, 2014.

WADA, Y. et al. Human water consumption intensifies hydrological drought worldwide. Environmental Research Letters, v.8, 2013.

WADDOCK, S. A.; GRAVES, S. B. The corporate social performance-financial performance link. Strategic Management Journal, v.18, p.303-19, 1997.

WOJDALSKI, J. et al. Determinants of water consumption in the dairy industry. Polish Journal of Chemical Technology, v.15, p.61-72, 2013. 
WOODSIDE, A. Case study research: theory, methods and practice. Bingley: Emerald Group Publishing, 2010. 455p.

WU, M. et al. Water consumption in the production of ethanol and petroleum gasoline. Environmental Management, v.44, p.981-97, 2009.

WU, Z.; WANG, Q. Effects of stage water shortage on water consumption and leaf area index of winter wheat. Nongye Gongcheng Xuebao/Transactions of the Chinese Society of Agricultural Engineering, v.26, p.63-8, 2010.

XIAO, H. Knowledge gaps on water issues and consumption habits in At-risk Chinese cities. International Journal of China Studies, v.4, p.327-41, 2013.

XIAO-JUN, W. et al. Climate change and water resources management in Tuwei river basin of Northwest China. Mitigation and Adaptation Strategies for Global Change, v.19, p.107-20, 2014.

XU, C.; SANO, M.; NAKATSUKA, T. A 400-year record of hydroclimate variability and local ENSO history in northern Southeast Asia inferred from tree-ring $8180 . \mathrm{Pa}$ laeogeography, Palaeoclimatology, Palaeoecology, v.386, p.588-98, 2013.

YIN, R. K. Applied social research methods series. 3. ed. Thousand Oaks: Sage, 2002. $192 p$.

. Case study research: design and methods. 4.ed. Thousand Oaks: SAGE Publications, 2008. 240p.

ZAIMES, G. N.; EMMANOULOUDIS, D. Sustainable management of the freshwater resources of Greece. Journal of Engineering Science and Technology Review, v.5, p.7782,2012 .

ZARZO, D.; CAMPOS, E.; TERRERO, P. Spanish experience in desalination for agriculture. Desalination and Water Treatment, v.51, p.53-66, 2013.

ZHANG, Q. et al. Influence of ENSO on precipitation in the East River basin, south China. Journal of Geophysical Research D: Atmospheres, v.118, p.2207-19, 2013.

RESUMO - Embora a crise no abastecimento de água na Região Metropolitana de São Paulo (RMSP) tenha se manifestado de maneira mais intensa no verão de 2013-2014, ela revela um problema crônico que vem afetando toda a Região nos últimos dez anos. Esse problema foi gerado pela falta de um planejamento estratégico que considere questões climatológicas que podem indicar, com meses de antecedência, problemas de recomposição dos níveis dos mananciais, permitindo que ações sejam empreendidas com razoável antecedência, reduzindo os impactos para a população. Este estudo mostra como é possível utilizar informações climáticas na gestão estratégica do sistema de abastecimento da RMSP.

PALAVRAS-CHAVE: Abastecimento urbano, Gestão de recursos hídricos, Água, São Paulo, Condições climáticas, Planejamento estratégico.

ABSTRACT - Though the crisis in the water supplying system in the Metropolitan Region of São Paulo (RMSP) was more intensively felt in the 2013-2014 summer, it reveals a 
chronic problem that has been affecting the whole RMSP for the past ten years. This problem is originated from the lack of a strategic planning that takes into consideration climate issues that could, months before, foresee problems to restore the levels of water resources, allowing measures to be implemented within a reasonable anticipation, therefore reducing the impacts on the population. This study shows how it is possible to use climate information in the strategic management of the water supply in the RMSP. KErWORDS: Urban water supply, Water resources management, Water, Sao Paulo, Weather conditions, Strategic planning.

Pedro Luiz Côrtes é professor-associado/livre-docente da Escola de Comunicações e Artes, da Universidade de São Paulo (ECA-USP). @ - plcortes@usp.br

Mauro Torrente é doutorando em Administração de Empresas do Programa de PósGraduação em Administração da Universidade Nove de Julho (Uninove).

@ - mrtorrente@gmail.com

Ailton Pinto Alves Filho é professor do Centro Universitário da FEI, São Bernardo do Campo, São Paulo. @ - ailtonline@gmail.com

Mauro Silva Ruiz é professor do Programa de Mestrado em Gestão Ambiental da Universidade Nove de Julho, São Paulo. @ - maurosilvaruiz@gmail.com

António José Guerner Dias é professor da Faculdade de Ciências da Universidade do Porto, Portugal. @-agdias@fc.up.pt

Rosely Rodrigues é mestre em Gestão Ambiental e Sustentabilidade pela Universidade Nove e Julho. @ - rosely.is.rodrigues@gmail.com

I Escola de Comunicações e Artes, Universidade de São Paulo. São Paulo/SP, Brasil.

II Programa de Pós-Graduação em Administração, Universidade Nove de Julho. São Paulo/SP, Brasil.

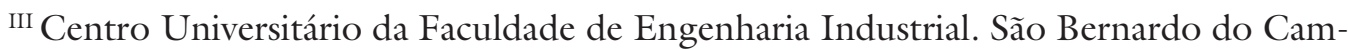
po/SP, Brasil.

Iv Programa de Pós-Graduação em Gestão Ambiental, Universidade Nove de Julho. São Paulo/SP, Brasil.

v Faculdade de Ciências da Universidade do Porto. Portugal.

VI Programa de Pós-Graduação em Gestão Ambiental e Sustentabilidade, Universidade Nove e Julho. São Paulo/SP, Brasil.

Recebido em 12.8.2014 e aprovado em 16.12.2014. 